\title{
microRNA-223 promotes the growth and invasion of glioblastoma cells by targeting tumor suppressor PAX6
}

\author{
BAI-SHENG HUANG ${ }^{1}$, QI-ZHI LUO ${ }^{2}$, YANG HAN ${ }^{1}$, XIAO-BO LI ${ }^{3}$, LI-JUN CAO ${ }^{4}$ and LI-XIANG WU ${ }^{1}$ \\ Departments of ${ }^{1}$ Physiology, and ${ }^{2}$ Immunology, Xiangya School of Medicine, Central South University, \\ Changsha 410078; ${ }^{3}$ Department of Neurology, Third Xiangya Hospital, Central South University, Changsha 410013; \\ ${ }^{4}$ Department of Anesthesiology, Second Xiangya Hospital, Central South University, Changsha 410015, P.R. China
}

Received June 21, 2013; Accepted July 29, 2013

DOI: $10.3892 /$ or.2013.2683

\begin{abstract}
Glioblastoma is the most common primary central nervous system malignancy and its unique invasiveness hinders effective treatment. Its high invasiveness may be controlled partly by microRNAs (miRNAs, miRs) and their target genes. In the present study, we found that increased miR-223 expression and reduced PAX6 expression coexisted in glioblastoma as detected by quantitative PCR or tissue microarrays. We confirmed that miR-223 directly targets PAX6 through binding to its 3'-UTR using dual luciferase reporter assay. In U251 and U373 glioblastoma cells, overexpression of miR-223 decreased PAX6 mRNA and protein expression; however, inhibition of miR-223 increased PAX6 mRNA and protein expression. Moreover, overexpression of miR-223 led to effects similar to those of PAX6 knockdown: increased cell viability, increased percentage of cells in the G1 phase and increased cell invasiveness parallel with increased MMP2, MMP9 and VEGFA expression. In addition, inhibition of miR-223 resulted in effects similar to those of PAX6 overexpression: decreased cell viability, decreased percentage of cells in the G1 phase and decreased cell invasiveness parallel with reduced MMP2, MMP9 and VEGFA expression. The data presented here suggest that miR-223 promotes the growth and invasion of U251 and U373 glioblastoma cells by targeting PAX6, which serves as a tumor suppressor in glioblastoma exerting the functions of inhibition of cell cycle transition, and the expression of MMP2, MMP9 and VEGFA. In conclusion, the present study supports miR-223 and PAX6 as novel therapeutic targets for glioblastoma.
\end{abstract}

\section{Introduction}

Glioblastoma is the most common primary central nervous system malignancy with features of localization in the brain,

Correspondence to: Professor Li-Xiang Wu, Department of Physiology, Xiangya School of Medicine, Central South University, 110 Xiangya Road, Hunan, Changsha 410078, P.R. China

E-mail:ywlx@mail.csu.edu.cn

Key words: glioblastoma, microRNAs, PAX6, invasion highly invasive behavior and extremely poor prognosis. The effects of current conventional therapeutic means for glioblastoma are not satisfactory (1). To improve the life expectancy and the quality of life of the patients, it is extremely critical to search for novel diagnostic and therapeutic targets. New hope for the treatment of human glioblastoma include microRNAs (miRNAs, miRs) that have recently emerged as key regulators of cancers (2). miRNAs, a class of small, non-coding, singlestranded RNAs, can post-transcriptionally regulate protein expression by targeting the 3'-untranslated region (3'-UTR) of target mRNAs, which cause either degradation or repression of translation. miRNAs are involved in multiple biological activities as well as progression of diseases including cancer (3). Many studies have shown that dysregulation of miRNAs plays an important role in the initiation and malignant progression of cancer (4-6).

miRNAs can act as either tumor suppressors or oncogenes depending on the functions of their targets. It has been reported that miR-223 functions as a tumor suppressor in certain types of cancers, for example, in the inhibition of osteosarcoma by targeting Hsp90B1 (7). miR-223 is weakly expressed in colorectal cancer, cervical cancer and hepatoma cells; and overexpression of miR-223 can suppress proliferation of the three types of tumor cells by targeting FOXO1 (8). Notably, miR-223 is high expressed in gastric cancer and promotes cellular proliferation and invasion by targeting EPB41L3 (9) or FBXW7/hCdc4 (10), functioning as an oncogene. In addition, miR-223 can target Stathmin1 which plays an oncogenic role in gastric cancer (11). Thus, the role of miR-223 may be opposing in different types of cancer or tumor cell lines via the targeting of different functional genes. Current studies have revealed that the growth and metastasis of glioblastoma are associated with various miRNAs (12-17). However, whether miR-223 serves as a tumor suppressor or an oncogene in glioblastoma remains unclear at present.

The paired box 6 (PAX6) encodes a transcription factor that is involved in the development of the brain, where its expression persists. It can suppress cell growth and invasion of glioblastoma cells, and has currently been demonstrated to function as a tumor suppressor in glioblastoma $(18,19)$. Yet, reports related to miRNAs of PAX6 are rare (20). Particularly, whether or not miR-223 targets PAX6 in glioblastoma requires elucidation. 
Therefore, in the present study, we determined the expression levels of miR-223 and PAX6 in glioblastoma cells or tissues. Then, the regulation of miR-223 on PAX6 expression and their effects on proliferation, cell cycle and invasion of glioblastoma U251 and U373 cells were investigated. It was confirmed that PAX6 is a target of miR-223 in glioblastoma. Our results may support miR-223 and/or PAX6 as novel diagnostic or therapeutic targets for glioblastoma.

\section{Materials and methods}

Cell culture. Glioblastoma A172, U251, U373 and U138 cell lines, and the human fetal glial HFGC cell line were purchased from the China Center for Type Culture Collection (CCTCC, Wuhan, China) and maintained in RPMI-1640 medium (HyClone) containing 10\% fetal bovine serum (Hangzhou Sijiqing) in a humidified atmosphere of $5 \% \mathrm{CO}_{2}$ at $37^{\circ} \mathrm{C}$.

Recombinant plasmid construction. To generate the PAX6overexpression plasmid, PAX6 CDS (NM_001258462) was amplified by PCR using the following primers: sense, 5 '-GGA TCCATGCAGAACAGTCACAGC-3' and antisense, 5'-GAA TTCTTACTGTAATCTTGGCCAGT-3', then the sequence was cloned into the $B a m \mathrm{HI} / E c o$ RI sites of the pcDNA3.1. The recombinant plasmid was termed pcDNA3.1-PAX6.

Transfection. For the miR-223 functional analysis, cells were transfected with the miR-223 scrambled miRNA control (negative control, NC), miR-223 mimics, or the miR-223 inhibitor (Ambion). To generate gain-of-function models of PAX6, cells were transfected with PAX6-pcDNA3.1 or its negative control (pcDNA3.1 vector), respectively. Transfection was performed using Lipofectamine 2000 (Invitrogen) according to the manufacturer's recommendations.

Lentivirus package and infection. The lentivirus system (Neuron, Shanghai, China) consisted of the pLKD-CMVG\&NR-U6-shRNA vector together with the plasmids containing the imperative elements for virus packaging. Short hairpin RNA (shRNA) targeting PAX6 (5'-ATGGGCGGAGT TATGATACCTAC-3') and the scrambled control (5'-AATTC TCCGAACGTGTCACGT-3') were coloned into the vector, respectively, which were then cotransfected with packaging plasmids into $293 \mathrm{~T}$ cells to generate individual lentiviruses. After $48 \mathrm{~h}$, the PAX6-RNAi-lentivirus and control lentivirus were harvested and purified. The lentivirus expressing PAX6 shRNA and the control lentivirus were termed Lv-PAX6shRNA and Lv-Ctr, respectively. U251 and U373 cells were plated in 6-well plates to reach $40-50 \%$ confluency. Appropriately titered lentivirus was added at a MOI of 20 and incubated at $37^{\circ} \mathrm{C}$.

Real-time RT-PCR. Total RNA was extracted from the cells with TRIzol reagent (Invitrogen) following the manufacturer's instructions. The relative expression level of miR-124 was determined by quantitative real-time RT-PCR using mirVana ${ }^{\mathrm{TM}}$ qRT-PCR microRNA detection kit (Ambion) following the manufacturer's instructions. Specific primer sets for miR-223 and U6 (used as an internal reference) were obtained from Ambion. Expression of PAX6 mRNA was detected by real- time RT-PCR using the standard SYBR-Green RT-PCR kit (Takara, Otsu, Japan) following the manufacturer's instructions. The specific primer pairs were as follows: PAX6 (157 bp) sense, 5'-AGACACAGCCCTCACAAAC-3' and antisense, 5'-ATCATAACTCCGCCCATTC-3'; $\beta$-actin (202 bp) as an internal control, sense, 5'-AGGGGCCGGACTCGTCATACT-3' and antisense, 5'-GGCGGCACCACCATGTACCCT-3'. The relative expression of PAX6 mRNA or miR-223 was quantified using the GraphPad Prism 4.0 software (GraphPad Software, San Diego, CA, USA) and the $2^{-\Delta \Delta \mathrm{Ct}}$ method (21).

Immunohistochemical analysis. A human brain gliocytoma and normal tissue microarray (Auragene Bioscience Co., Changsha, China) containing 5 cases of normal tissue, 5 cases of T1N0M0 glioblastoma, 4 cases of T2N0M0 glioblastoma and other subtypes of brain gliocytoma (data not shown), duplicate cores per case, was processed for immunohistochemical analysis using anti-PAX6 antibody (Millipore) as previously described (22). Images were analyzed using Leica Q550 image analysis system (Leica, Wetzlar, Germany). The mean optical density value (D) and area (A) of brown particles in three visual fields of each section were calculated by the system. The expression levels of PAX6 in tissues were evaluated using the formula: integral density $=\mathrm{D} \times \mathrm{A}$.

Dual luciferase reporter assay. The 3'-UTR of PAX6 (NM_000280) containing the miRNA-223 binding sites and its corresponding mutated sequence were cloned into the psiCHECK2 luciferase reporter vector (Promega) downstream of Renilla luciferase, named PAX6 3'-UTR-psi-CHECK2 and PAX6 Mut 3'-UTR-psi-CHECK2, respectively. Using Lipofectamine 2000 (Invitrogen), U251 and U373 cells were co-transfected with the reporter constructs and miR-223 mimics, the miR-223 inhibitor, negative control (NC) or the negative control inhibitor. Luciferase activity was determined after $48 \mathrm{~h}$ using the Dual-Glo substrate system (Promega) and a Beckman Coulter LD400 luminometer. Data are presented as the ratio of experimental (Renilla) luciferase to control (Firefly) luciferase.

Cell proliferation assay. For analysis of the functions of miR-223, U251 and U373 cells were transfected with miR-223 mimics or its inhibitor, respectively. For analysis of PAX6 functions, U251 and U373 cells were either transfected with pcDNA3.1-PAX6 or infected with Lv-PAX6-shRNA. Cells in exponential growth were plated at a final concentration of $2 \times 10^{3}$ cells/well in 96 -well plates. The viability of the cells was evaluated by MTT assay after 24, 48, 72 and $96 \mathrm{~h}$ of seeding. The optical density at $570 \mathrm{~nm}$ (OD570) of each well was measured with an ELISA reader (ELX-800 type, Bio-Tek).

Flow cytometric (FCM) analysis. The cells were digested and collected after $48 \mathrm{~h}$ post-transfection or $96 \mathrm{~h}$ post-infection, and washed with PBS twice. The cells were resuspended in PBS and then fixed in $70 \%$ ethanol at $4^{\circ} \mathrm{C}$ for $18 \mathrm{~h}$. The cells were washed with PBS and resuspended in staining solution ( $50 \mu \mathrm{g} / \mathrm{ml}$ of propidium iodide, $1 \mathrm{mg} / \mathrm{ml}$ of RNase A, $0.1 \%$ Triton X-100 in PBS). The stained cells $\left(1 \times 10^{5}\right)$ were then analyzed with a flow cytometer (Beckman Coulter). 
A

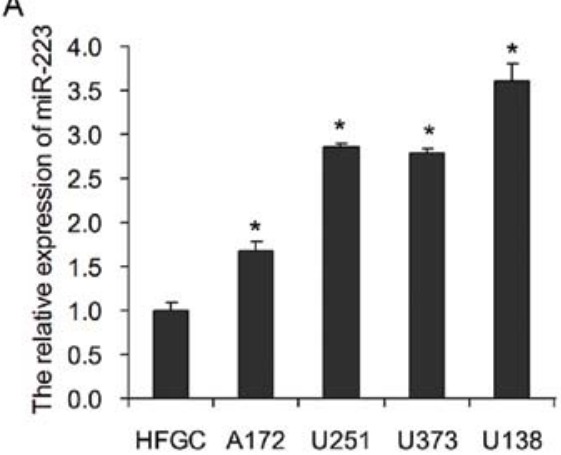

B

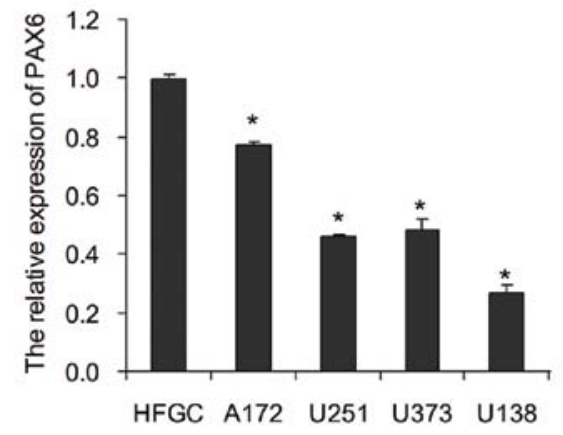

C

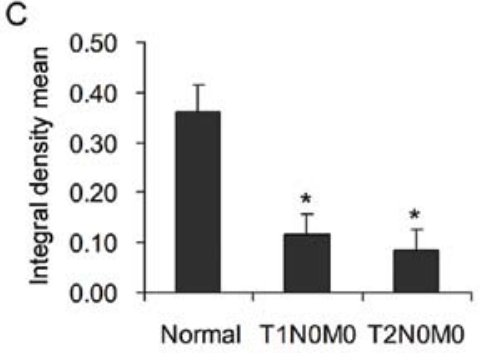

D

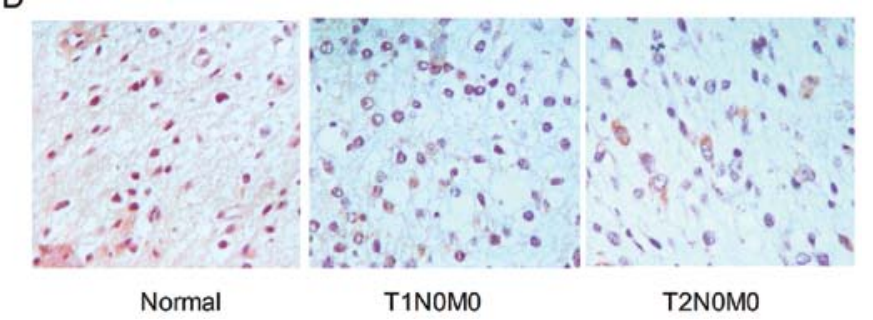

Figure 1. Expression of miR-223 and PAX6 in glioblastoma. (A) Increased miR-223 and (B) decreased PAX6 was detected in human glioblastoma A172, U251, U373 and U138 cells, when compared to the expression level in human fetal glial HFGC cells, using qRT-PCR. (C) PAX6 protein expression (presented as integral density) was decreased in grade T1N0M0 and T2N0M0 human glioblastoma tissues, when compared to the expression in the normal brain tissues, using a tissue microarray as detected by immunohistochemical analysis. (D) Representative results of PAX6 expression (brown particles) in glioblastoma tissues. ${ }^{\mathrm{P}}<0.05$ vs. (A and B) HFGC cells or (C) the normal group.

Cell invasion assay. The cell invasion assay was performed using a Cell Invasion Assay kit (Chemicon International, Temecula, CA, USA) according to the manufacturer's guidelines. Briefly, U251 or U373 cells were placed in the upper compartment of the chambers, and RPMI-1640 containing $10 \%$ fetal bovine serum was added to the lower chambers. After $24 \mathrm{~h}$ of incubation at $37^{\circ} \mathrm{C}$, cells on the upper face of the membrane were scraped using a cotton swab and cells on the lower face were fixed, stained and observed under a microscope. The dye on the membrane was then dissolved with $10 \%$ acetic acid, dispensed into 96-well plates (150 $\mu \mathrm{l} /$ well), and the optical density at $570 \mathrm{~nm}$ (OD570) of each well was measured with an ELISA reader (ELX-800 type; Bio-Tek).

Western blotting. Cells were lysed in cell lysate, and then centrifuged at $12,000 \mathrm{x}$ g for $20 \mathrm{~min}$ at $4^{\circ} \mathrm{C}$. The supernatant was collected and denatured. Proteins were separated using $10 \%$ SDS-PAGE and blotted onto a polyvinylidene difluoride membrane (PVDF). The PVDF membrane was treated with TBST containing $50 \mathrm{~g} / \mathrm{l}$ skimmed milk at room temperature for $4 \mathrm{~h}$, followed by incubation with the primary antibodies anti-PAX6, anti-MMP-2, anti-MMP-9, anti-VEGFA (Abcam) and anti- $\beta$-actin (Abzoom, Dallas, TX, USA), respectively, at $37^{\circ} \mathrm{C}$ for $1 \mathrm{~h}$. Membranes were rinsed and incubated for $1 \mathrm{~h}$ with the corresponding peroxidase-conjugated secondary antibodies. Chemiluminent detection was performed with the ECL kit (Pierce Chemical, Rockford, IL, USA). The amount of the protein of interest, expressed as arbitrary densitometric units, was normalized to the densitometric units of $\beta$-actin.

Statistical analysis. Data are expressed as the means \pm SD from at least three separate experiments. Statistical analysis was carried out using SPSS 15.0 software. The difference between two groups was analyzed by the Student's t-test. A value of $\mathrm{P}<0.05$ was considered to indicate a statistically significant result.

\section{Results}

Expression of miR-223 and PAX6 in glioblastoma. The expression levels of miR-223 and PAX6 in human glioblastoma A172, U251, U373 and U138 cells were determined using quantitative RT-PCR (qRT-PCR). Compared to the human fetal glial HFGC cell line, the miR-223 expression level was apparently increased in the glioblastoma cells (Fig. 1A); however, the PAX6 expression level was markedly decreased (Fig. 1B). Moreover, we found that the PAX6 protein was weakly expressed in the glioblastoma tissues (Fig. 1C and D), using the glioblastoma tissue microarrays. These results suggest that miR-223 and PAX6 are potential regulatory molecules in the progression of glioblastoma, and PAX6 is a potential diagnostic biomarker for glioblastoma.

PAX6 is a direct target of miR-223. To confirm whether PAX6 is a direct target of miR-223, we subcloned the PAX6 3'-UTR fragment containing the miR-223 binding site and mutated targeting sequence, and cloned them into psi-CHECK2 dual luciferase reporter vectors. The miR-223 mimics significantly inhibited the luciferase activity in the HEK293T cells transfected with the PAX6 3'-UTR-psi-CHECK2 reporter vector (Fig. 2A). However, miR-223 mimics did not suppress the luciferase activity levels in the HEK293T cells transfected with the PAX6 Mut 3'-UTR-psi-CHECK2 reporter vector (Fig. 2B). Thus, PAX6 was confirmed as a direct target of miR-223. 

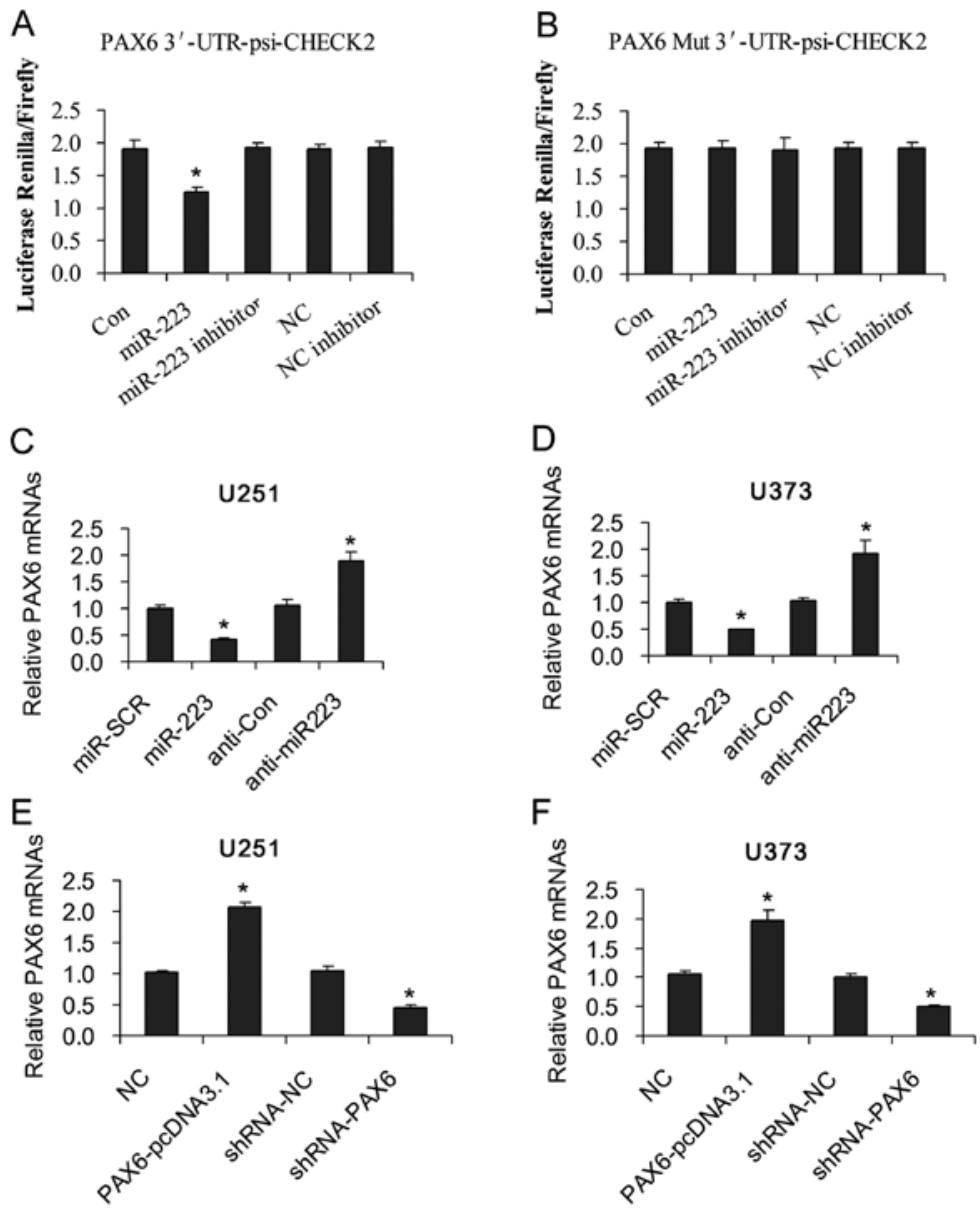

Figure 2. miR-223 targeting of PAX6 and regulation of PAX6 expression. Dual luciferase reporter assays were performed to examine the interaction of miR-223 and its targeting sequence in the PAX6 3'-UTR using constructs containing (A) the targeting sequence (PAX6 3'-UTR-psi-CHECK2 reporter vector) and the (B) mutated targeting sequence (PAX6 Mut 3'-UTR-psi-CHECK2 reporter vector). The relative PAX6 mRNA levels in (C) U251 and (D) U373 cells transfected with miR-223 mimics or miR-223 inhibitor (anti-miR-223), as detected by qRT-PCR. The relative PAX6 mRNA levels in (E) U251 and (F) U373 cells after transfection of the PAX6-pcDNA3.1 construct or infection of the Lv-shRNA-PAX6, as detected by qRT-PCR. " $\mathrm{P}<0.05$ vs. (A and B) Con, (C and D) miR-SCR or (E and F) NC.

To further investigate the regulation of PAX6 by miR-223 in glioblastoma cells, the expression levels of PAX6 mRNA in the U251 and U373 cells transfected with miR-223 mimics or the miR-223 inhibitor (anti-miR-223) were detected. The results showed that the introduction of miR-223 mimics to glioblastoma U251 and U373 cells decreased PAX6 mRNA expression. Moreover, the miR-223 inhibitor increased the PAX6 mRNA expression levels (Fig. 2C and D). This indicates that miR-223 functions as a suppressor of PAX6 by targeting PAX6 in glioblastoma cells.

PAX6 inhibits the growth and invasion of glioblastoma cells. To investigate the roles of PAX6 in the glioblastoma cells, PAX6 was overexpressed or knocked down in the U251 and U373 cells by transfection of the PAX6-pcDNA3.1 construct or infection of Lv-shRNA-PAX6, respectively (Fig. 2E and F). The data showed that overexpression of PAX6 decreased the cell viability (Fig. 3A and B), the percentage of cells in the G1 phase (Fig. 3C-F) and cell invasiveness (Fig. 3G and H) of the U251 and U373 cells; and knockdown of PAX6 caused a reverse result. This suggests that PAX6, which serves as a tumor suppressor, plays an important role in the control of growth and metastasis of glioblastoma.
miR-223 promotes the growth and invasion of glioblastoma cells. Based on the above data, we hypothesized that miR-223 is also able to affect the growth and invasion of glioblastoma cells by regulation of PAX6 expression. Our results showed that introduction of miR-223 mimics to U251 and U373 cells increased their cell viability (Fig. 4A and B), the percentage of cells in the G1 phase (Fig. 4C-F) and cell invasiveness (Fig. 4G and $\mathrm{H}$ ); however, introduction of the miR-223 inhibitor (antimiR-223) to both cell lines decreased their cell viability, the percentage of cells in the G1 phase and cell invasiveness. This suggests that miR-223, which functions as an oncogene, plays a role in the regulation of growth and metastasis of glioblastoma; and its underlying mechanisms may include its targeted regulation of PAX6 expression.

Effects of PAX6 and miR-223 on MMP2, MMP9 and VEGFA expression. Western blot analysis showed that overexpression of PAX6 resulted in the downregulation of MMP2, MMP9 and VEGFA, and knockdown of PAX6 caused upregulation of MMP2, MMP9 and VEGFA in glioblastoma U251 (Fig. 5A) and U373 (Fig. 5B) cells. Correspondingly, introduction of miR-223 mimics to glioblastoma U251 and U373 cells led to effects similar to those following knockdown of PAX6 in 

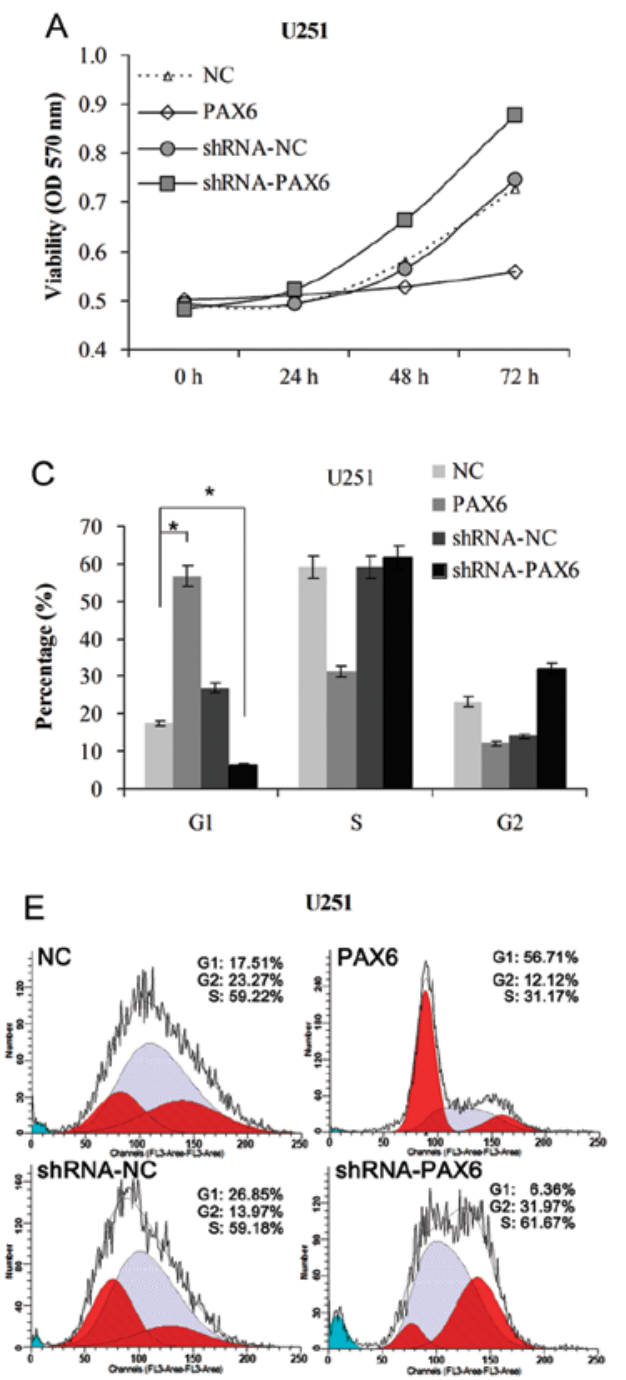
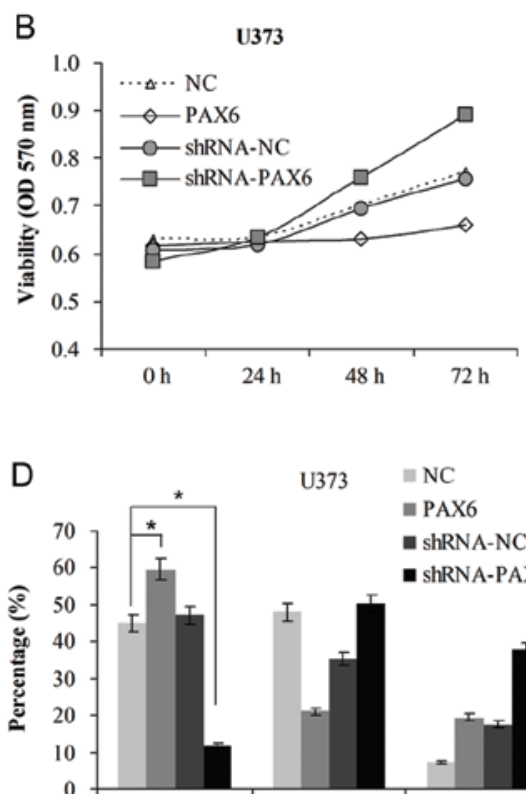

G1

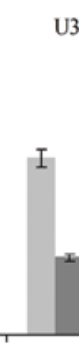

F
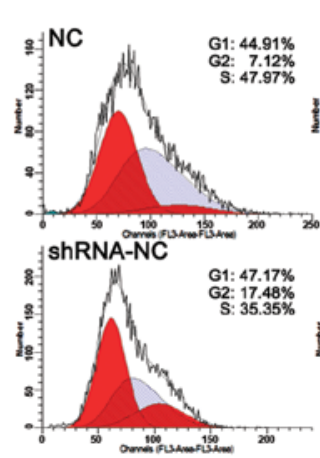

$373=\mathrm{NC}$

= PAX6

G2
-

- shRNA-PAX6
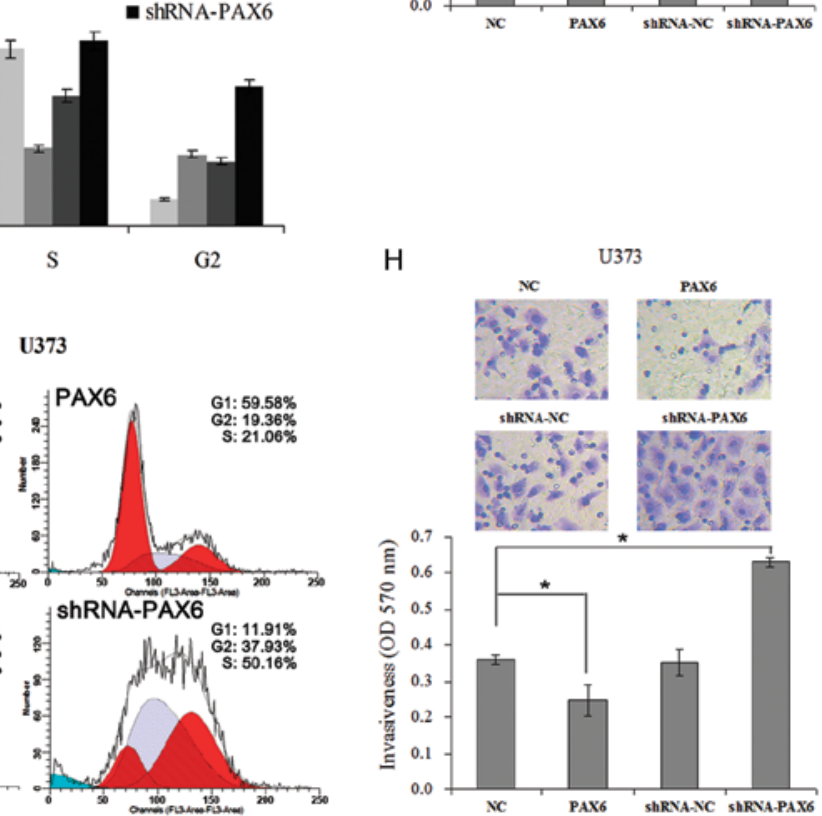

Figure 3. PAX6 inhibits the growth and invasion of glioblastoma cells. U251 and U373 cells were transfected with the PAX6-pcDNA3.1 construct (PAX6) or infected with the Lv-shRNA-PAX6 (shRNA-PAX6). PAX6 inhibited the cell viability of (A) U251 and (B) U373 cells, as determined by MTT assay. PAX6 increased the percentages of G1 phase cells in the (C and E) U251 and (D and F) U373 cell lines, as determined by FCM analysis. PAX6 inhibited the invasion of (G) U251 and (H) U373 cells, as determined by Transwell assay. " $\mathrm{P}<0.05$.

both cell lines: enhanced MMP2, MMP9 and VEGFA expression. Introduction of the miR-223 inhibitor resulted in effects similar to those following overexpression of PAX6 in both cell lines: decreased MMP2, MMP9 and VEGFA expression. This demonstrates that the miR-223-mediated process of the promotion of growth and metastasis of glioblastoma possibly occur through targeting PAX6 and regulating MMP2, MMP9 and VEGFA expression.

\section{Discussion}

Cancer initiation and progression are associated with abnormal expression of various genes or miRNAs. Identification of dysregulated miRNAs and their target genes is important for elucidating molecular mechanisms of cancer pathology and discovering novel diagnostic and therapeutic targets. Studies indicate that miR-223 functions as an oncogene or a tumor suppressor in cancer related to the cancer type (7-11). Our results showed that the miR-223 expression level in the human glioblastoma A172, U251, U373 and U138 cells was higher than that in human fetal glial HFGC cells, indicating that miR-223 is increased in glioblastoma. It has been reported that PAX6 regulates growth, invasion and angiogenesis in multiple types of cancer including glioblastoma $(15,18,19,23)$. We found that the PAX6 expression level was decreased in the glioblastoma A172, U251, U373 and U138 cells and tumor tissues from patients with glioblastoma. This demonstrated that increased miR-223 expression and reduced PAX6 expression coexist in glioblastoma, implying a potential correlation between them.

We next confirmed that miR-223 directly targets PAX6 through binding to its 3'-UTR using a dual luciferase reporter assay. In addition, post-transcriptional regulation of PAX6 expression by miR-223 was observed in glioblastoma U251 and U373 cells. In the glioblastoma cells, upregulation of miR-223 decreased PAX6 mRNA and protein expression; however, inhibition of miR-223 increased PAX6 mRNA and protein expression. This suggests that PAX6 is a direct target of miR-223 in glioblastoma. To investigate their functional correlations, we analyzed the effects of PAX6 and miR-223 on the growth, invasion and the related signaling molecules of 
A

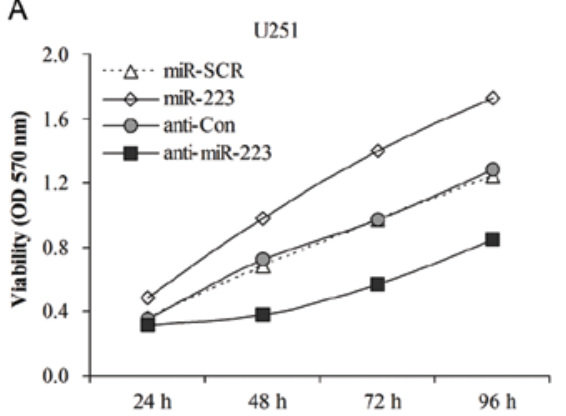

C

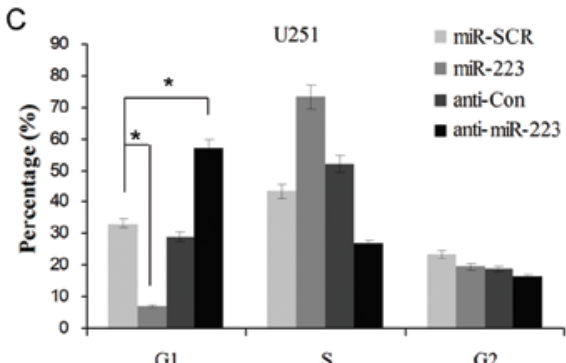

$E$

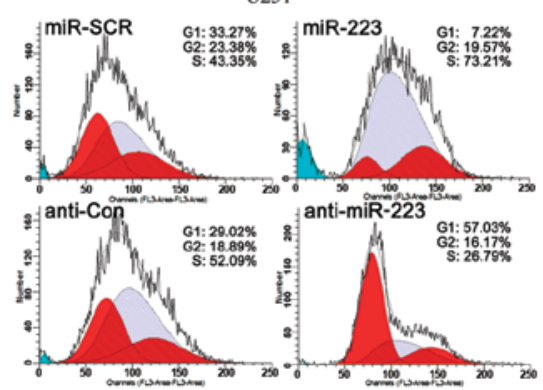

B

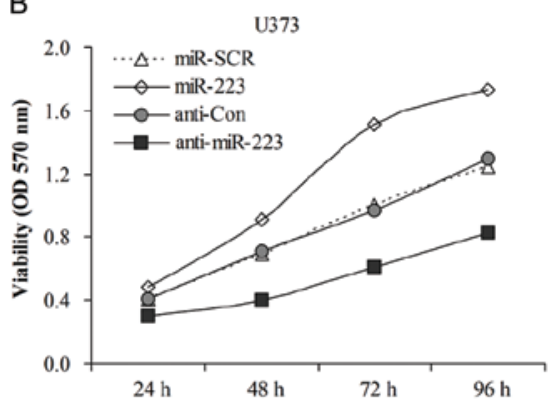

D

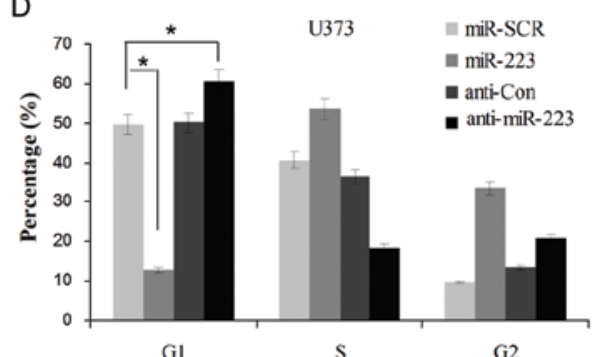

$\mathrm{F}$

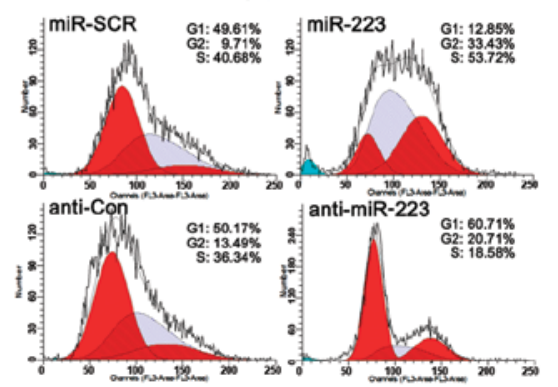

G
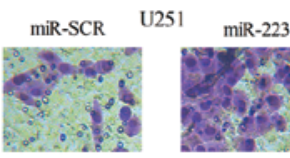

anti-Con
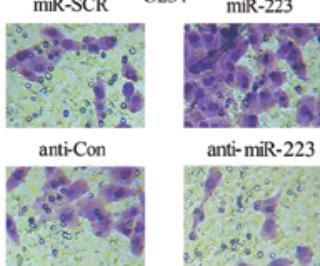

anti-miR-223

$20^{\circ} 0^{\circ}$

58
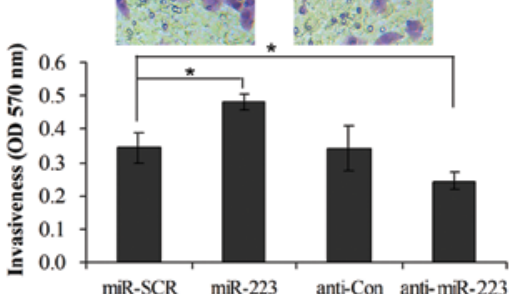

miR-SCR miR-223 anti-Con anti-miR-223

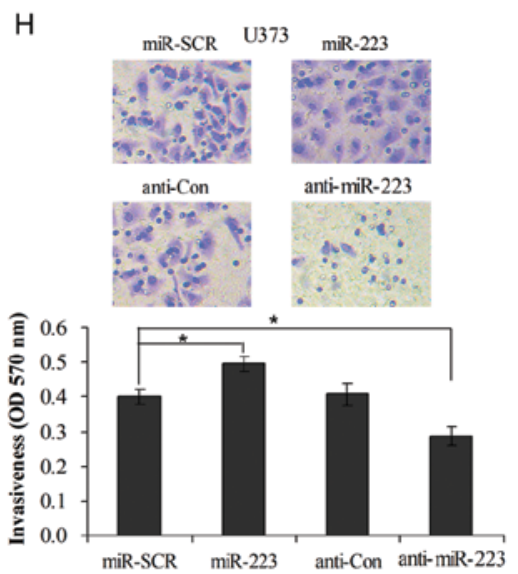

Figure 4. miR-223 promotes the growth and invasion of glioblastoma cells. U251 and U373 cells were transfected with miR-223 mimics or the miR-223 inhibitor (anti-miR-223). miR-223 increased the cell viability of (A) U251 and (B) U373, as determined by the MTT assay. miR-223 decreased the percentages of G1 phase cells in (C and E) U251 and (D and F) U373 cell lines, as determined by FCM analysis. PAX6 promoted the invasion of (G) U251 and (H) U373 cells, as determined by Transwell assay. ${ }^{*} \mathrm{P}<0.05$.

A

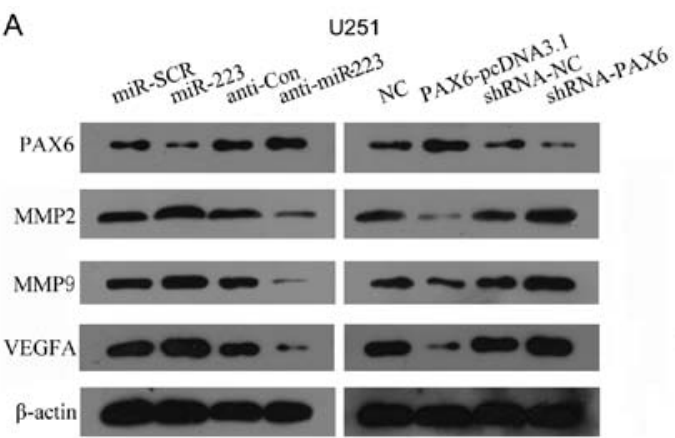

B

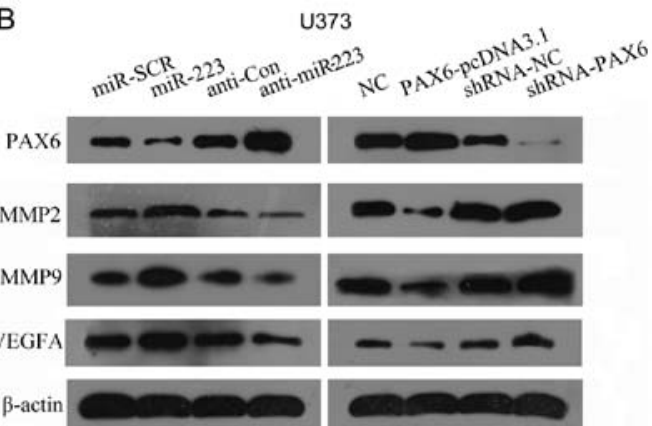

Figure 5. Effects of PAX6 and miR-223 on MMP2, MMP9 and VEGFA expression. The expression levels of PAX6, MMP2, MMP9 and VEGFA in (A) U251 and (B) U373 cells transfected with PAX6-pcDNA3.1 construct (PAX6), infected with Lv-shRNA-PAX6 (shRNA-PAX6), or transfected with miR-223 mimics or the miR-223 inhibitor (anti-miR-223) were detected by western blot analysis.

glioblastoma cells. In glioblastoma U251 and U373 cells, overexpression of miR-223 led to effects similar to those following knockdown of PAX6: increased cell viability, percentage of cells in the G1 phase and cell invasiveness parallel to increased MMP2, MMP9 and VEGFA expression. Moreover, inhibition of miR-223 resulted in effects similar to those following overexpression of PAX6: decreased cell viability, percentage of cells in the G1 phase and cell invasiveness parallel to reduced MMP2, MMP9 and VEGFA expression. These results indicate that miR-223 functions as an oncogene in glioblastoma and the underlying mechanisms include its targeting of PAX6, which serves as a tumor suppressor in glioblastoma.

It is worth noting that PAX6 is also considered an oncogene promoting proliferation and tumorigenesis in some types of 
cancers such as human retinoblastoma (24) and in breast cancer cells (25). However, in the present study, we demonstrated that PAX6 functions as a tumor suppressor in glioblastoma cells, and our conclusion is in accordance with the results obtained by Mayes et al (18) and Chang et al (26). PAX6, as a transcription factor, suppresses cell growth by inhibition of G1/S transition (19) and decreases invasiveness by inhibiting the expression of MMP2, MMP9 and VEGFA possibly through its direct binding to their promoters $(18,23,27)$. Cell migration and invasion are initial steps in cancer metastasis. PAX6 downregulation facilitates enhancement of MMP2 and MMP9 expression, promoting glioblastoma cell enzymatic degradation of the extracellular matrix and increase in its invasive phenotype (28). Vascular endothelial growth factors (VEGF) play important roles in angiogenesis. VEGFA is frequently overexpressed in cancers and its overexpression promotes cancer vasculogenesis, growth and metastasis $(29,30)$. Therefore, PAX6 inhibits the growth and invasion of glioblastoma by suppression of its cell cycle transition and the expression of MMP2, MMP9 and VEGFA. Correspondingly, miR-223 promotes proliferation, cell cycle transition and invasion of glioblastoma cells possibly by downregulation of PAX6 expression.

Although our data confirm that miR-223 functions as an oncogenic miRNA in glioblastoma cells by its targeting of PAX6, whether PAX6 is regulated by other miRNAs [for example, miR-328 (20) or miR-7a (31)] or whether miR-223 also targets other downstream genes [for example, EPB41L3 (9) or FBXW7/hCdc4 (10)] in glioblastoma warrants further investigation.

In brief, PAX6 is weakly expressed in glioblastoma parallel to increased miR-223 expression, and is a target of miR-223. miR-223 can promote growth and invasion of glioblastoma U251 and U373 cells by targeting PAX6, which serves as a tumor suppressor in glioblastoma exerting the functions of inhibition of cell cycle progression, and the expression of MMP2, MMP9 and VEGFA. Collectively, the present study suggests that miR-223 and PAX6 are novel therapeutic targets for glioblastoma.

\section{Acknowledgements}

The present study was supported by the National Natural Science Foundation of China (30770838).

\section{References}

1. Preusser M, de Ribaupierre S, Wohrer A, et al: Current concepts and management of glioblastoma. Ann Neurol 70: 9-21, 2011.

2. Qu S, Yao Y, Shang C, et al: MicroRNA-330 is an oncogenic factor in glioblastoma cells by regulating SH3GL2 gene. PloS One 7: e46010, 2012.

3. Lages E, Ipas H, Guttin A, Nesr H, Berger F and Issartel JP: MicroRNAs: molecular features and role in cancer. Front Biosci 17: 2508-2540, 2012.

4. Chang HT, Li SC, Ho MR, et al: Comprehensive analysis of microRNAs in breast cancer. BMC Genomics 13 (Suppl 7): S18, 2012.

5. Chen PS, Su JL and Hung MC: Dysregulation of microRNAs in cancer. J Biomed Sci 19: 90, 2012.

6. Liu H: MicroRNAs in breast cancer initiation and progression. Cell Mol Life Sci 69: 3587-3599, 2012.
7. Li G, Cai M, Fu D, et al: Heat shock protein 90B1 plays an oncogenic role and is a target of microRNA-223 in human osteosarcoma. Cell Physiol Biochem 30: 1481-1490, 2012.

8. Wu L, Li H, Jia CY, et al: MicroRNA-223 regulates FOXO1 expression and cell proliferation. FEBS Lett 586: 1038-1043, 2012.

9. Li X, Zhang Y, Zhang H, et al: miRNA-223 promotes gastric cancer invasion and metastasis by targeting tumor suppressor EPB41L3. Mol Cancer Res 9: 824-833, 2011.

10. Li J, Guo Y, Liang X, et al: MicroRNA-223 functions as an oncogene in human gastric cancer by targeting FBXW7/hCdc4. J Cancer Res Clin Oncol 138: 763-774, 2012.

11. Kang W, Tong JH, Chan AW, et al: Stathmin1 plays oncogenic role and is a target of microRNA-223 in gastric cancer. PloS One 7: e33919, 2012.

12. Zhao S, Deng Y, Liu Y, et al: MicroRNA-153 is tumor suppressive in glioblastoma stem cells. Mol Biol Rep 40: 2789-2798, 2013.

13. Zhang QQ, Xu H, Huang MB, et al: MicroRNA-195 plays a tumor-suppressor role in human glioblastoma cells by targeting signaling pathways involved in cellular proliferation and invasion. Neuro Oncol 14: 278-287, 2012.

14. Yue X, Wang P, Xu J, et al: MicroRNA-205 functions as a tumor suppressor in human glioblastoma cells by targeting VEGF-A. Oncol Rep 27: 1200-1206, 2012.

15. Lin J, Teo S, Lam DH, Jeyaseelan K and Wang S: MicroRNA-10b pleiotropically regulates invasion, angiogenicity and apoptosis of tumor cells resembling mesenchymal subtype of glioblastoma multiforme. Cell Death Dis 3: e398, 2012.

16. Fang X, Yoon JG, Li L, et al: The SOX2 response program in glioblastoma multiforme: an integrated ChIP-seq, expression microarray, and microRNA analysis. BMC Genomics 12: 11, 2011.

17. Li Y, Guessous F, Zhang Y, et al: MicroRNA-34a inhibits glioblastoma growth by targeting multiple oncogenes. Cancer Res 69: 7569-7576, 2009

18. Mayes DA, Hu Y, Teng Y, et al: PAX6 suppresses the invasiveness of glioblastoma cells and the expression of the matrix metalloproteinase-2 gene. Cancer Res 66: 9809-9817, 2006.

19. Zhou YH, Wu X, Tan F, et al: PAX6 suppresses growth of human glioblastoma cells. J Neurooncol 71: 223-229, 2005.

20. Chen KC, Hsi E, Hu CY, Chou WW, Liang CL and Juo SH: MicroRNA-328 may influence myopia development by mediating the PAX6 gene. Invest Ophthalmol Vis Sci 53: 2732-2739, 2012.

21. Arocho A, Chen B, Ladanyi M and Pan Q: Validation of the 2-DeltaDeltaCt calculation as an alternate method of data analysis for quantitative PCR of BCR-ABL P210 transcripts. Diagn Mol Pathol 15: 56-61, 2006.

22. Jiang $X$, Yue J, Lu H, et al: Inhibition of filamin-A reduces cancer metastatic potential. Int J Biol Sci 9: 67-77, 2013.

23. Zhou YH, Hu Y, Mayes D, et al: PAX6 suppression of glioma angiogenesis and the expression of vascular endothelial growth factor A. J Neurooncol 96: 191-200, 2010.

24. Li L, Li B, Zhang H, et al: Lentiviral vector-mediated PAX6 overexpression promotes growth and inhibits apoptosis of human retinoblastoma cells. Invest Ophthalmol Vis Sci 52: 8393-8400, 2011.

25. Zong X, Yang H, Yu Y, et al: Possible role of Pax-6 in promoting breast cancer cell proliferation and tumorigenesis. BMB Rep 44: 595-600, 2011.

26. Chang JY, Hu Y, Siegel E, Stanley L and Zhou YH: PAX6 increases glioma cell susceptibility to detachment and oxidative stress. J Neurooncol 84: 9-19, 2007.

27. Sivak JM, West-Mays JA, Yee A, Williams T and Fini ME: Transcription factors Pax6 and AP- $2 \alpha$ interact to coordinate corneal epithelial repair by controlling expression of matrix metalloproteinase gelatinase B. Mol Cell Biol 24: 245-257, 2004.

28. Zhang W, Fulci G, Buhrman JS, et al: Bevacizumab with angiostatin-armed oHSV increases antiangiogenesis and decreases bevacizumab-induced invasion in U87 glioma. Mol Ther 20: 37-45, 2012.

29. Qiu JF, Zhang ZQ, Wang Y and You J: Lentivirus-mediated RNAi knockdown of VEGFA in RKO colorectal cancer cells decreases tumor formation and growth in vitro and in vivo. Int $\mathrm{J}$ Clin Exp Pathol 5: 290-298, 2012.

30. Chen CH, Lai JM, Chou TY, et al: VEGFA upregulates FLJ10540 and modulates migration and invasion of lung cancer via PI3K/ AKT pathway. PloS One 4: e5052, 2009.

31. de Chevigny A, Core N, Follert P, et al: miR-7a regulation of Pax6 controls spatial origin of forebrain dopaminergic neurons. Nat Neurosci 15: 1120-1126, 2012. 